\title{
ITEM D
}

Dissertações e Teses 


\section{DISSERTAÇÕES DE MESTRADO}

001 ALEXANDRE, Gisela Angelina Levatti (1982) Hidrogeoquimica das águas do Rio Paraguai em Corumbá (Estado de Mato Grosso do Sul); caracteristicas físicas, físico-químicas e químicas. $87 \mathrm{p}$.

Orientadora: Mária Szikszay.

002 AMARAL, Ivan Amorosino do (1982) O conteudo e o enfoque dos livros de geologia introdutória. 259p.

Orientador: Franco Levi.

003 ARRUDA, Josué Rabelo (1977) Estudos em modelos reduzidos de estruturas geológicas por eletrorresistitividade. $81 \mathrm{p}$.

Orientador: André Davino.

004 ARTUR, Antonio Carlos (1980) Rochas metamórficas dos arredores de Itapira, SP. 193p. Orientador: Koji Kawashita.

005 BANDEIRA Jr., Alfredo Nunes (1977) Sedimentologia e microfácies calcáreas das Formações Riachuelo e Cotinguiba da Bacia Sergipe/Alagoas. 59p.

Orientador: Kenitiro Suguio.

006 BARRIOS, Fernando José (1983) A caracterização geocronológica da região amazônica da Venezuela. $123 \mathrm{p}$.

Orientador: Umberto Giuseppe Cordani.

007 BASEI, Miguel Ângelo Stipp (1977) Idade do vulcanismo ácido intermediário na Regiāo Amazônica. 133p.

Orientador: Umberto Giuseppe Cordani.

008 BELLO, Rosa Maria da Silveira (1978) Condições de metamorfismo de Buritirama, Pará e Serra do Navio, Amapá. 154p.

Orientador: José Vicente Valarelli.

009 BRANDT NETO, Max (1977) Estratigrafia da Formação Bauru na região do baixo Tietê. 74p. Orientador: Setembrino Petri.

010 CAETANO, Maria Rita (1978) Aplicação de métodos quantitativos ao estudo comparado de seç̧ões litológicas do Paleozóico Superior da bacia sedimentar do Paraná (Grupos Tubarão e Passa Dois). 88 p.

Orientador: Paulo Milton Barbosa Landim.

011 CAMPANHA, Ginaldo Ademar da Cruz (1981) O lineamento de Além Paraiba na área de Três Rios, RJ. 109 p.

Orientador: Fernando Flávio Marques de Almeida.

012 CARNEIRO, Celso Dal Ré (1977) Geologia e evolução geológica da Folha de São José dos Campos, $S P .106 \mathrm{p}$.

Orientador: Yociteru Hasui.

013 CARVALHO, Sebastião Gomes de (1983) Geologia e potencial de mineralização dos arredores de Fortaleza de Minas, MG. 130p.

Orientador: Aledir Paganelli Barbour.

014 COELHO, Paulino Eduardo Fernandes Pinto (1980) Reconhecimento radiogeológico da região de Perús, SP. $104 \mathrm{p}$.

Orientador: Aledir Paganelli Barbour.

015 COTTAS, Luiz Roberto (1977) Geologia da área de Nuporanga-Batatais, SP - uma contribuição ao estudo do Cenozóico Paulista. 143p.

Orientador: Vicente José Fúlfaro. 
FERREIRA, Francisco José Fonseca (1983) Integração de dados aeromagnéticos e geológicos: configuração e evolução tectônica do Arco de Ponta Grossa. 169p.

Orientador: André Davino.

017 FIORI, Alberto Pio (1977) Estratigrafia do Grupo Tubarão (Formação Aquidauana) na região sudoeste do Estado de Minas Gerais. 86p.

Orientador: Paulo Milton Barbosa Landim.

018 FITTIPALDI, Fernando Cilento (1982) A caracterização cuticular de glossopteris communisfeistmantel, 1876, Formação Rio Bonito (Permiano Inferior), na Bacia do Paraná, Brasil. 76p. Orientador: Oscar Rösler.

019 FRAZÃO, Ely Borges (1981) Contribuição à metodologia para determinação da pressão de expansão em solos e rochas. $71 \mathrm{p}$.

Orientador: José Eduardo Siqueira Farjallat.

020 GONÇALVES, Neide Maria Malusá (1979) Estudo dos materiais superficiais da região de Ribeirão Preto, SP, e suas relações com elementos morfológicos da paisagem. $177 \mathrm{p}$.

Orientador: Adolpho José Melfi.

021 GROKE, Maria Cristina Toledo (1981) Evolução geoquímica, mineralógica e micromorfológica no processo de bauxitização. Estudo comparativo dos depósitos de Mogi das Cruzes, Curucutu e Lavrinhas, SP, associados a diferentes tipos litológicos. 106p.

Orientador: Adolpho José Melfi.

022 IWANUCH, Woldemar (1982) Geologia da região do Domo do Sucunduri, 149p.

Orientador: Umberto Giuseppe Cordani.

023 JARDIM, Niza Silva (1979) Estado atual da documentação geológica brasileira - proposta de uma linguagem de indexação (Thesaurus) para o tratamento da produção bibliográfica. $141 \mathrm{p}$. Orientador: André Davino.

024 KRAUSPENHAR, Élida Brum (1977) Aplicação de microcospia eletrônica ao estudo dos sedimentos cenozóicos da Provincia Costeira do Rio Grande do Sul, Brasil. 114p. Orientador: Antonio Carlos Rocha Campos.

025 MACEDO, Arlei Benedito (1980) Estudo estatistico das relações entre elementos principais e traços em rochas basálticas da Bacia do Paraná. $171 \mathrm{p}$.

Orientador: Nabor Ricardo Rüegg.

026 MACHADO, Rômulo (1977) Geologia e gênese do depósito de manganês de Bandarra, Município de Jacaraci, Bahia. 109p.

Orientador: Evaristo Ribeiro Filho.

027 MENDES, José Milton Benetti (1980) Prospecção de calcário por métodos geofísicos em Matosinhos, MG, Geologia - Eletrorresistividade. 41p.

Orientador: André Davino.

028 MOÇO, Moacyr Francisco (1981) Prospeç̧ão de mármore para cantaria em Quixabá, Municipio de Pio IX, PI. 144p.

Orientador: Reinholt Ellert.

029 NEGRÃO, Oscar Braz Mendonza (1983) A pesquisa e a metodologia de ensino nas Escolas Superiores de Graduação em Geologia no país. 186p.

Orientador: Franco Levi.

030 OLIVEIRA, Antonio Manoel dos Santos (1981) Estudo da percolação d'água em maciços rochosos para o projeto de grandes barragens. $197 \mathrm{p}$.

Orientador: José Eduardo Siqueira Farjallat.

031 ORSATTI, Wanderley (1979) Disjunções colunares e direções de corridas de Lava. 68p. Orientador: José Vicente Valarelli.

032 PANIAGUA, Remy David Antezana (1977) Importância da estação sismográfica tipo arranjo de Valinhos. $115 \mathrm{p}$.

Orientador: Evaristo Ribeiro Filho.

033 PARISOT, Elisabeth Hillairet (1983) As águas subterrâneas no Centro Oeste do Municipio de São Paulo - características hidrogeológicas e quimicas. 93p.

Orientador: Aldo da Cunha Rebouças. 

Alto Seridó, com ênfase às suas manifestaçōes nos Plagioclásios dos Orto-Anfibolitos. 48p. Orientador: José Vicente Valarelli.

035 RAGONHA, Evaldo Wehmuth (1978) Chondrichthyes do Membro Taquaral (Formação Irati) no Estado de São Paulo, 65p.

Orientador: Rubens da Silva Santos.

036 RATTI, Giuliana (1980) Avaliação de ataques químicos para determinações geoquímicas por espectrofotometria de absorção atômica. $134 \mathrm{p}$.

Orientador: José Vicente Valarelli.

037 REIS NETO, José Manoel dos (1983) Evolução geotectônica da bacia do Alto Tocantins, Goiás. 98p.

Orientador: Umberto Giuseppe Cordani.

038 RUBERTI, Excelso (1977) Mineralogia quimica dos anfibolitos da regiấo de Morretes-Antonina, Paraná, 86p.

Orientador: Celso de Barros Gomes.

039 SAAD, Antonio Roberto (1977) Estratigrafia do Subgrupo Itararé no Centro Sul do Estado de São Paulo. $107 \mathrm{p}$.

Orientador: Antonio Carlos Rocha Campos.

040 SANTOS, Paulo Roberto dos (1979) Distribuição estratigráfica, caracteristica e fácies de diamictitos e rochas associadas do Subgrupo Itararé no Centro e Sul do Estado de São Paulo. 135p. Orientador: Antonio Carlos Rocha Campos.

041 SCHEVICW, Flavia Goulart Pucci (1980)Alteração intempérica dos termos ultramáficos do complexo de Piên, PR, estudo mineralógico-geoquímico. 172p.

Orientador: Jean Jacques Pierre Trescases.

042 SÍGOLO, Joel Barbujiani (1979) Geologia de depósitos residuais bauxiticos na regiâo de Lavrinhas, $S P$, e sua viabilidade econômica. 190p.

Orientador: Evaristo Ribeiro Filho.

043 SILVA, Antonio Carlos Gondim de Andrade E (1980) Mineralogia, material amorfo, evolução genética e estudo do niquel e cromo no perfil de alteração do maciço ultramáfico de Santa Fé, Goiás. 136p.

Orientador: Aledir Paganelli Barbour.

044 SILVA, José Renato Morando da (1978) Composição isotópica de Carbono e Oxigênio na determinação de paleoambientes. 59p.

Orientador: Kenitiro Suguio.

045 SILVA, Marcos Egydio da (1981) Análise estrutural das faixas ectiniticas associadas ao falhamento de Cubatão entre as região de Juquia e Pedro Barros, Estado de São Paulo. 118p.

Orientador: Georg Robert Sadowski.

046 SOARES, Lindolfo (1982) O emprego de diafragma plástico na recuperação de barragens. ${ }_{107 \mathrm{p}}$ Orientador: José Eduardo Siqueira Farjallat.

047 SUSLICK, Saul Barisnik (1978) Estudo metodológico de técnicas estatisticas para análise de dados geoquimicos. 116p.

Orientador: Gilberto Amaral.

048 TAI, Wu Fu (1981) Minerais pesados das seqüências arenosas paleozóica e mesozóica no Centro Leste do Estado de São Paulo. 78p.

Orientador: Paulo Milton Barbosa Landim.

049 TASSINARI, Colombo Celso Gaeta (1981) Evolução geotectônica da Provincia Rio Negro-Juruena na região amazônica. 99p.

Orientador: Koji Kawashita.

050 TEIXEIRA, Wilson (1978) Significação tectônica do magmatismo anorogênico básico e alcalino na Amazônia. 99p.

Orientador: Umberto Giuseppe Cordani. 
051 TESSLER, Moysés Gonzalez (1982) Sedimentação atual na Regiāo Lagunar de Cananéia - Iguape, Estado de São Paulo. 169p.

Orientador: Kenitiro Suguio.

052 WINTERS, Andreas Antonius Maria (1982) A geologia do maciço sienitico da Pedra Branca, Caldas, MG. 92p.

Orientador: José Moacyr Vianna Coutinho.

053 ZAINE, Mariselma Ferreira (1980) Uma barreira geográfica no Paleozóico Superior na região de Fartura, SP. 89p.

Orientador: Vicente José Fúlfaro.

\section{TESES DE DOUTORAMENTO}

054 BARCELOS, José Humberto (1979) Arenitos cretácicos do alto Parnaiba, MG: proveniência e ambientes de sedimentação. $181 \mathrm{p}$.

Orientador: Kenitiro Suguio.

055 BITTENCOURT, Andre Virmond Lima (1978) Sólidos hidrotransportados na bacia hidrográfica do Rio Ivai: aplicação de balanços hidrogeoquimicos na compreensão de evolução dos processos da dinâmica externa. 201p.

Orientador: Adolpho José Melfi.

056 CAMPOS, Jayme de Oliveira (1981)

Propriedades geotécnicas e comportamento tecnológico de arenitos da Formação Caiuá. $251 \mathrm{p}$.

Orientador: José Eduardo Siqueira Farjallat.

057 CANDIA, Maria Ângela Fornoni (1983) Petrologia dos complexos máfico-ultramáficos de Mangabal I e Mangabal II, Sanclerlândia, Goiás. 400p.

Orientador: Vicente Antonio Vitório Girardi.

058 CERRI, Carlos Clemente (1979) Alteração e pedogênese em areas graniticas situadas sob diferentes condições bioclimáticas encontradas no Brasil. $241 \mathrm{p}$.

Orientador: Adolpho José Melfi.

059 COIMBRA, Armando Márcio (1983) Estudo sedimentológico e geoquímico do Permo-Triássico da bacia do Maranhão. 259p.

Orientador: Setembrino Petri.

060 DUARTE, Uriel (1980) Geologia ambiental. 73p.

Orientador: Nelson Ellert.

061 FIORI, Alberto Pio (1979) Geologia da região de Pouso Alegre - Machado: análise estrutural de dobramentos superpostos. 200p.

Orientador: Paulo Milton Barbosa Landim.

062 FURTADO, Valdenir Veronese (1979) Contribuição ao estudo da sedimentaçāo atual no canal de São Sebastião, Estado de São Paulo. 110p.

Orientador: Kenitiro Suguio.

063 GAMA JR., Ercilio Gonzaga da (1977) Sistemas deposicionais e modelo de sedimentação das Formações Campos e Emboré, Bacia de Campos, Rio de Janeiro, Brasil. 104p.

Orientador: Kenitiro Suguio.

064 HARALYI, Nicolau Ladislau Ervin (1979) Carta gravimétrica do oeste de Minas Gerais, sudeste de Goiás e norte de São Paulo. 152p.

Orientador: André Davino.

065 KIHARA, Yushiro (1983) O estudo mineralógico das cinzas volantes brasileiras: origem, caracteristicas e qualidade. $164 \mathrm{p}$.

Orientador: José Moacyr Vianna Coutinho.

066 LIMA, Murilo Rodolfo de (1978) Palinologia da Formação Santana (Cretáceo do nordeste do Brasil). 335p.

Orientador: Oscar Rosler. 
067 MACIEL FILHO, Carlos Leite (1983) Estudo do processo geoquímico de obstrução de filtro de barragens. $136 \mathrm{p}$.

Orientador: Sérgio Estanislau do Amaral

068 MADUREIRA FILHO, José Barbosa de (1983) Determinação física da composição molecular de Granadas. 181p.

Orientador: William Gerson Rolim de Camargo.

069 MELLO, Everaldo Zeferino Vieira de (1982) Estudo da ocorrência de fluorita, barita e metais básicos da Fazenda Jurema, Barra da Estiva, Bahia. 221p.

Orientador: Reinholt Ellert.

070 MUSSA, Diana (1982) Lignitafofloras permianas da Bacia do Paraná, Brasil (Estados de São Paulo e Santa Catarina). 553p.

Orientador: Antonio Carlos Rocha Campos.

071 OLIVEIRA, Mary Elizabeth Cerruti Bernardes de (1977) Tafoflora eogondvânica da camada Irapuá, Formação Rio Bonito (Grupo Tubarão), SC. 301p.

Orientador: Josué Camargo Mendes.

072 OLIVEIRA, Sônia Maria Barros de (1980) Alteraçāo intempérica das rochas ultrabásicas de Santa Fé, GO, e gênese do depósito niquelifero. 216p.

Orientador: Adolpho José Melfi.

073 PARADELA, Waldir Renato (1983) Discriminação de unidades litológicas no baixo vale do Rio Curuçá (Bahia) através de realces por processamento digital de dados MSS-Landsat 3. 249p. Orientador: Gilberto Amaral.

074 ROSA, Rosa Beatriz Gouvêa da Silva (1983) Estudo hidroquimico e isotópico das águas subterrâneas do Aquifero Botucatu no Estado de São Paulo. 133p. Orientador: Aldo da Cunha Rebouças.

075 SÁ, José Haroldo da Silva (1978) Pegmatitos litiniferos da região de Itinga-Araçuai, Minas Gerais. 104p. Orientador: Reinholt Ellert.

076 SARTORI, Pedro Luiz Pretz (1978) Petrologia do Complexo Granitico de São Sepé, RS: modelo evolucional de granitos do sul do Brasil. 196p.

Orientador: Nabor Ricardo Rüegg.

077 SEIGNEMARTIN, Claudio Lisias (1979) Geologia de áreas urbanas: o exemplo de Ribeirão Preto, SP. $126 \mathrm{p}$.

Orientador: Vicente José Fúlfaro.

078 SOUZA, José Vitorino de (1979) Geologia e gênese do protominério e do minério da província manganesifera de Araçoiaba-Pacajús, no Estado do Ceará. 167p.

Orientador: Evaristo Ribeiro Filho.

\section{TESES DE LIVRE-DOCENCIA}

BRITO NEVES, Benjamin Bley (1983) O mapa geológico do Nordeste Oriental do Brasil, escala 1/1.000.000. $177 \mathrm{p}$.

080 HYPÓLITO, Raphael (1980) Criptomelana - sintese e estabilidade. 160p.

081 SADOWSKI, Georg Robert (1983) Sobre a geologia estrutural de cinturões de cisalhamento continentais.

082 SCHULTZ-GÜTTLER, Rainer Aloys (1982) Os carbonatos de cálcio, manganês, magnésio e ferro: um modelo da energia de mistura. 109p.

083 SVISERO, Darcy Pedro (1978) Composição quimica, origem e significado geológico de inclusões minerais de diamantes do Brasil. 156p.

084 SZIKSZAY, Mária (1981) Hidrogeoquímica das fontes de Äguas da Prata. 2v. (193 e 98p.) 